\title{
Development of the Polystyrene Scintillator Technology and Particle Detectors on Their Base
}

\author{
Vladimir Rykalin, Valery Brekhovskikh, Sergey Chernichenko, AlexandreGorin and Vitaliy Semenov \\ State Research Center of Russian Federation, Institute for High Energy Physics, Protvino142281, Russia
}

\begin{abstract}
The new designed at Institute for High Energy Physics (IHEP) technologies of polystyrene scintillators manufacturing, molding of polystyrene granules under pressure, melting of granules in forms with mirrors walls, production of scintillating granules and detectors on their basis are considered. The main characteristics of the polystyrene scintillators produced by a method of large-block polymerization and a method of extruding of scintillation strips from polymerized blocks are provided in this article also.
\end{abstract}

Key words: Polystyrene scintillators, melting technology, "bulk" type counters.

\section{Nomenclature}

$\begin{array}{ll}\text { E: } & \text { Energy }(\mathrm{GeV}) \\ \text { EM: } & \text { Electromagnetic } \\ \text { ph.e.: } & \text { Photoelectrons } \\ \text { PMT: } & \text { Photomultiplier } \\ \text { MIP: } & \text { Minimal ionization particle } \\ \text { SNM: } & \text { Special Nuclear Materials } \\ \text { WLS: } & \text { Wave Length Shifter } \\ \text { TNF } & \text { Tagged Neutrino Facility }\end{array}$

\section{Greek Letter}

$\sigma . \quad$ Resolution

\section{Introduction}

The manufacture of polystyrene scintillators was started at IHEP about 30 years ago. The large scintillation block thermal polymerization technology followed by machining and polishing use at that time. The next step was the development and introduction of new technology, highly transparent scintillator strips extruded from large-block scintillators. The molding under pressure machines and the industrial extruder of the IHEP scintillator facility equipped with modern industrial machines, allows to produce scintillators commercially starts in early 2000s. Highly transparent

Corresponding author: Vladimir Rykalin, Ph.D., professor, research fields: particles physics and quantum engineering. E-mail: Vladimir.Rykalin@ihep.ru. detectors on the base of this scintillation strips and plates manufactured by the technology of WLS-fibers light collection have been widely used in a number of experiments, both at the IHEP accelerator, and the physical facilities overseas centers. Others high-technology methods for scintillator manufacturing developed at the IHEP to date are briefly described in this article.

\section{IHEP Polystyrene Scintillator Facility}

\subsection{Large Blocks of Scintillators Produced by Polymerizing}

Scintillators production started in IHEP about 30 years ago. Initially, it was machining of large thermally polymerized blocks. Some characteristics of the developed scintillators are provided in Tables 1 and 2.

Some samples of the polymerized scintillators are shown in Fig. 1.

The extrusion technology of bars and rods up to 5 meters length from large-size polymerized scintillator blocks was developed afterwards [1]. High volume transparency (about $2 \mathrm{~m}$ ) of extruded scintillator (cross-section up to $10 \times 250 \mathrm{~mm}^{2}$ ) allowed to use them in experiments at IHEP: in the hodoscope hadrons detector of general algebraic modeling system (GAMS) [2], in the Large Aperture Muon Hodoscopes 
Table 1 The main characteristics of the developed scintillators.

\begin{tabular}{ll}
\hline Main characteristics & Forms and sizes \\
\hline Base: polystyrene & Scintillators can be produced as bars, sheets, blocks, rods. Bars can \\
Density: $1.05 \mathrm{~g} / \mathrm{cm}^{3}$ & reach 4m length at width up to $250 \mathrm{~mm}^{3}$ and thickness up to $25 \mathrm{~mm}$. \\
Coefficient of refraction: 1.59 & Blocks can reach $500 \times 250 \times 250 \mathrm{~mm}^{3}$ \\
Ratio H/C: 1 & \\
Solution: dissolved in toluene, ethers ... & \\
\hline
\end{tabular}

Table 2 Physical characteristics (bulk scintillators) (http://exwww.ihep.ru/scint/bulk/product.htm).

\begin{tabular}{|c|c|c|c|c|c|}
\hline $\begin{array}{l}\text { Type of a } \\
\text { scintillator }\end{array}$ & $\begin{array}{l}\text { Light output from } \\
\text { anthracene (\%) }\end{array}$ & $\begin{array}{l}\text { Time decay } \\
(\mathrm{ns})\end{array}$ & $\begin{array}{l}\text { Maximum of irradiation } \\
\text { spectra }(\mathrm{nm})\end{array}$ & $\begin{array}{l}\text { Attenuation length } \\
(\mathrm{cm})^{*}\end{array}$ & Main applications \\
\hline SC-201 & 55 & 3.0 & 420 & 200 & $\beta, \gamma, \alpha$, fast $n$ \\
\hline SC-202 & 52 & 4.6 & 465 & 200 & $\beta, \gamma, \alpha$, fast $n$ \\
\hline SC-203 & 52 & 4.5 & 480 & $>200$ & $\beta, \gamma, \alpha$, fast $n$ \\
\hline SC-204 & 52 & 5.0 & 490 & $>200$ & $\beta, \gamma, \alpha$, fast $n$ \\
\hline SC-205 & 66 & 2.4 & 420 & 200 & $\beta, \gamma, \alpha$, fast $n$ \\
\hline SC-206 & 67 & 1.8 & 430 & 200 & $\beta, \gamma, \alpha$, fast $n$ \\
\hline SC-207 & 44 & 1.8 & 380 & 50 & For time of flight detectors \\
\hline SC-221 & 32 & 2.4 & 430 & 50 & $1.7 \% \mathrm{Sn} ;$ for low energy $\gamma$ \\
\hline SC-222 & 59 & 2.4 & 430 & 50 & $1.7 \% \mathrm{Sn}$; for low energy $\gamma$ \\
\hline SC-223 & 52 & 2.4 & 430 & 50 & $2.4 \% \mathrm{~Pb}$; for low energy $\gamma$ \\
\hline SC-231 & 56 & 2.4 & 430 & 100 & $\begin{array}{l}0.6 \%{ }^{10} \mathrm{~B} \text {; for } n \text { of thermal } \\
\text { energy }\end{array}$ \\
\hline
\end{tabular}

* Attenuation length for samples of $1 \times 20 \times 200 \mathrm{~cm}^{3}$ with polished surface.

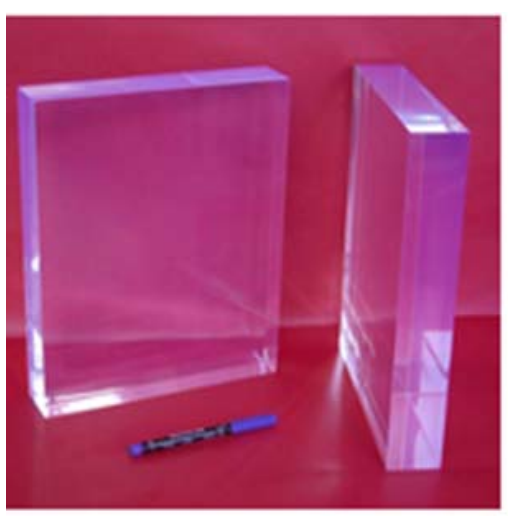

(a)

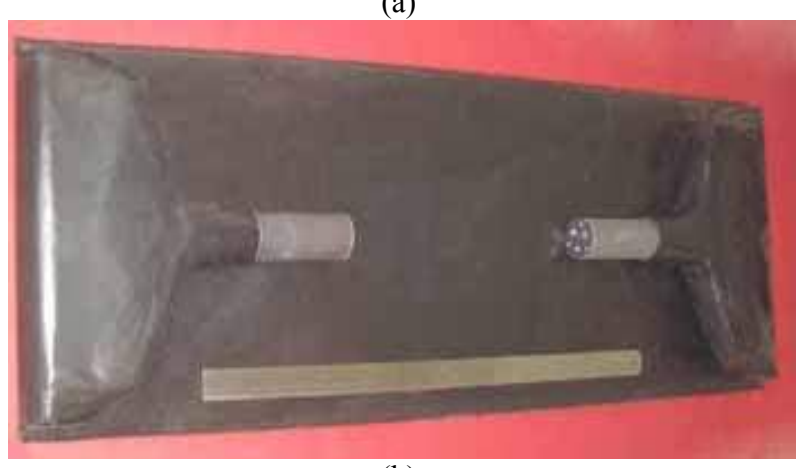

(b)

Fig. 1 (a) Scintillating plates and parallelepiped; (b) scintillating counter with the scintillator of $10 \times 500 \times 2,000$ $\mathbf{m m}^{3}$ sizes for scintillating detector planes without empty gaps. of TNF [3] and at CERN: in the Large Aperture Hodoscopes of NA-12 [4], in the Forward Muon Hodoscope and Shower Maximum Detector of DELPHI [5, 6], in the Muon Hodoscope of DIRAC [7].

\subsection{Application of the Industrial Methods of Scintillators Manufacture}

The manufacturing process of scintillators on the base of a molding under pressure was used at IHEP in the late 1980s at IHEP [8, 9]. It was proposed for the first time to use commercial polystyrene granules powdered by scintillation additives for the producing of scintillators using a molding under pressure. They have been designed for use with WLS-fiber readout. That made the requirement for a long attenuation in scintillator less important.

Plates and short strips manufacturing at IHEP with molding under pressure technology are the highest demand now. These plates are using in several detectors in high energy physics (HEP) experimental setups on particle accelerators (electromagnetic and hadron calorimeters, guard counters) and for detecting 
cosmic showers (large area detectors with a fiber readout).

The molding under pressure machines and the industrial extruder of the IHEP scintillator facility put into operation in early 2000s are shown at Fig. 2.

This large-tonnage technology has provided the possibility to create cellular wide aperture electromagnetic [10] and hadron calorimeters [11-14]. Cells were fabricated as metal-scintillator "sandwich" modules. The thickness of the scintillator variation $1-15 \mathrm{~mm}$ and the transverse dimensions of a square are 40-200 $\mathrm{mm}$. The production of injection moldings plates with different configurations including grooves and holes for WLS-fibers (Figs. 3-5) was setting up in IHEP for the mass production of a wide range of scintillator plates.

The physical characteristics of the developed scintillators are provided in Table 3.

The total number of counters with more than $1 \times 1$ $\mathrm{m}^{2}$ square [15], assembled from the injection moldingscintillatorswith the dimensions $200 \times 200 \times$ $5 \mathrm{~mm}^{3}$ manufactured for different laboratories is about 500. 1.5 tons of similar elements, but with the element dimensions $\left(120 \times 100 \times 5 \mathrm{~mm}^{3}\right)$, shown in Fig. 3, have been used in guard systems (SNM monitors) [16]. Typical number of PMT photoelectrons for the assembly of two plates $(10 \mathrm{~mm}$ thick) is 40 ph.e./MeV.

Electromagnetic calorimeter of "Shashlyk" type with optimal configuration on the base of injection molding scintillator was designed at IHEP in the early 2000s and tested jointly with INR RAS group is shown in Figs. 4 and 5 [17-20]. After that, "Shashlyk"began to be widely used [21-23].

The modules with a record for this type of calorimeter energy resolution $\sigma(E) / E=4 \% / \sqrt{E}$ are presented in Fig. 6. Module size is $110 \times 110 \times$ $650 \mathrm{~mm}^{3}$ and sampling structure is about 300 layers of $1.5 \mathrm{~mm}$ scintillator plates and $0.275 \mathrm{~mm}$ lead.

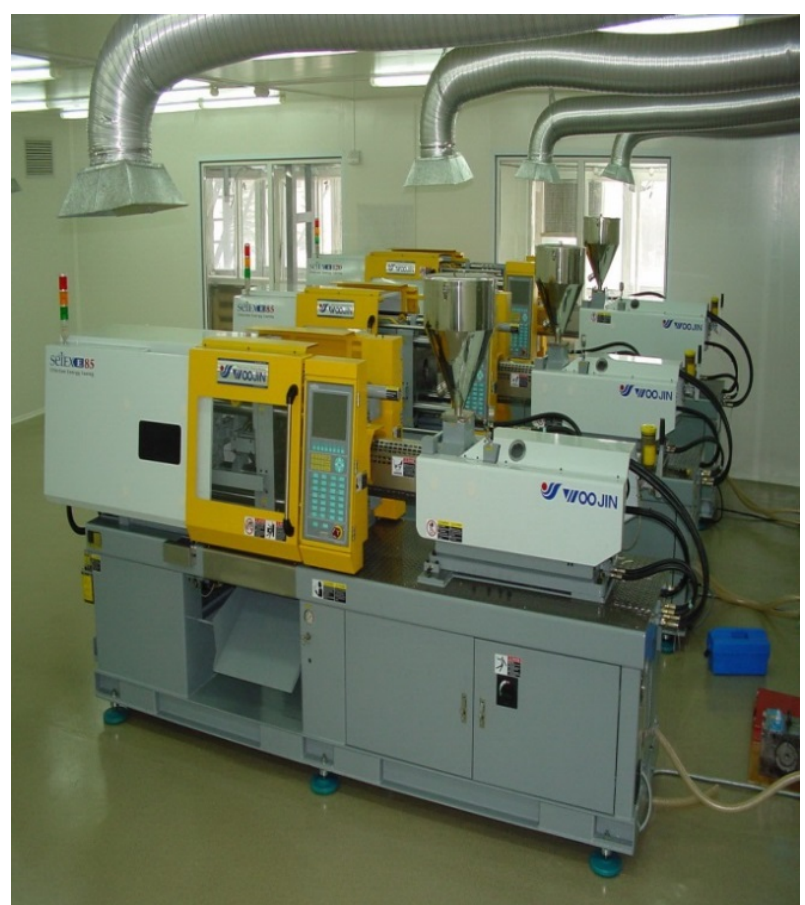

(a)

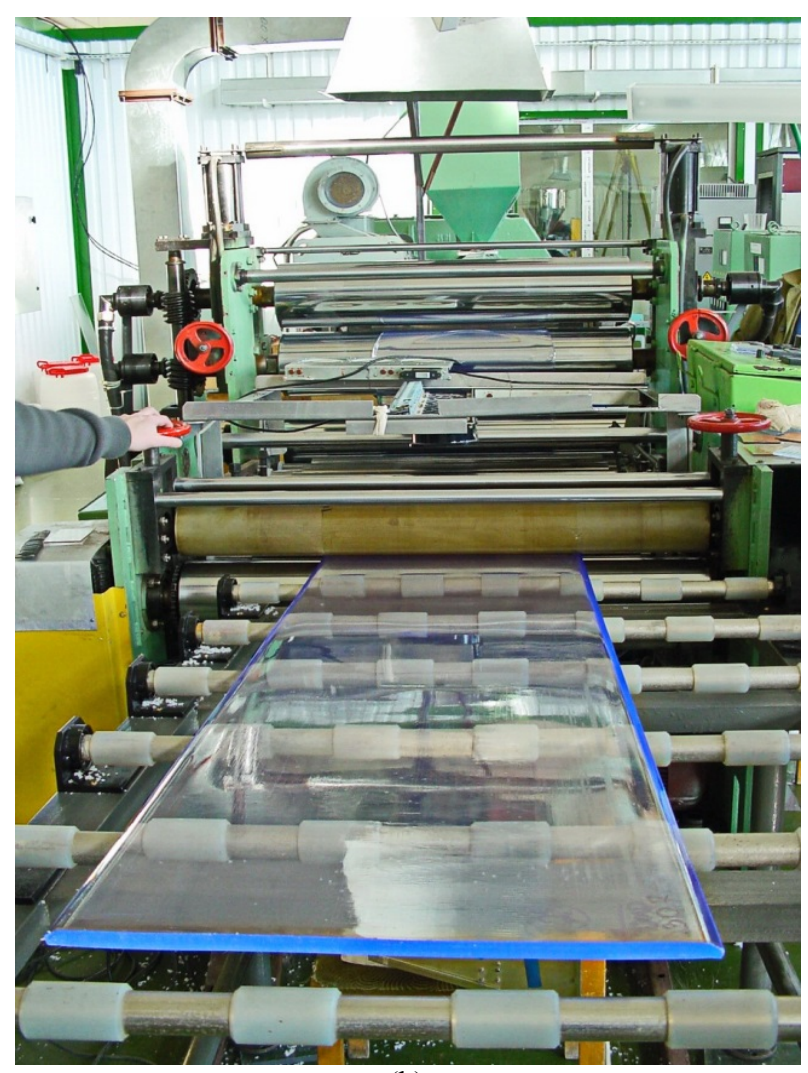

(b)

Fig. 2 (a) IHEP molding under pressure machines; (b) industrial extruder for production of scintillating bars and strips. 

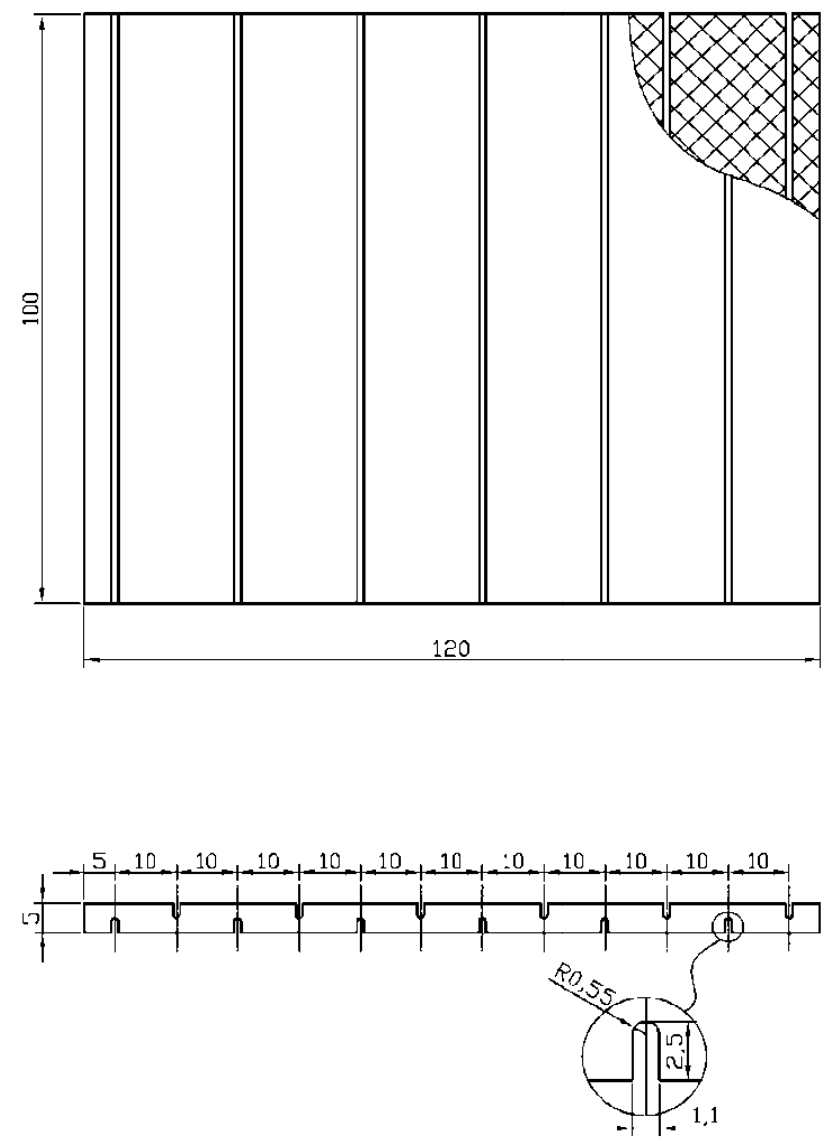

Fig. 3 Scintillating element of large sizes counters with WLS light collection.

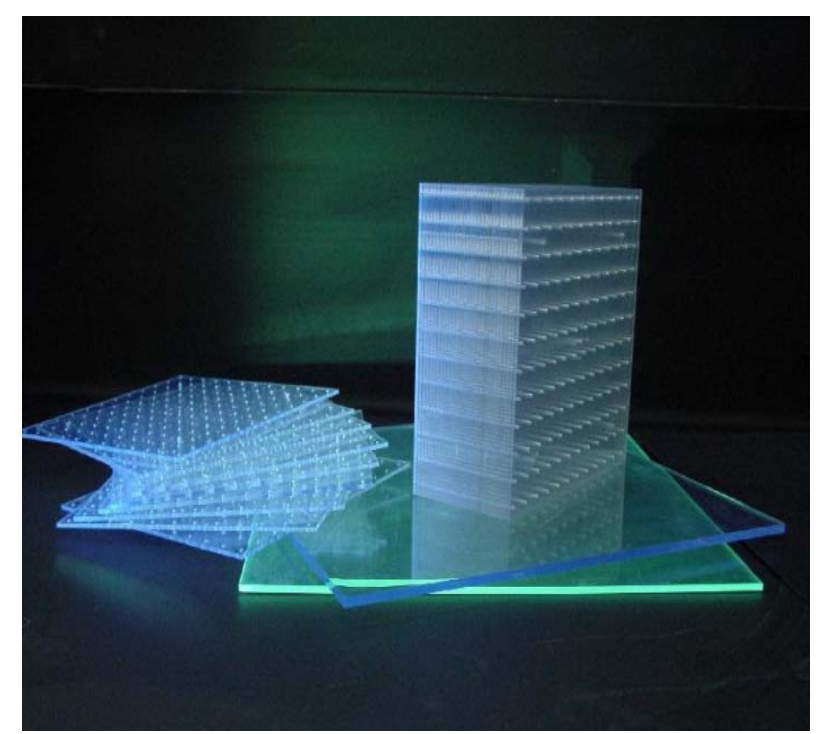

Fig. 4 Photos of scintillation plates of different sizes and different configuration producing by the injection molding.
Fig. 5 Scintillating element of electromagnetic calorimeter (Shashlyk type) with WLS light collection.

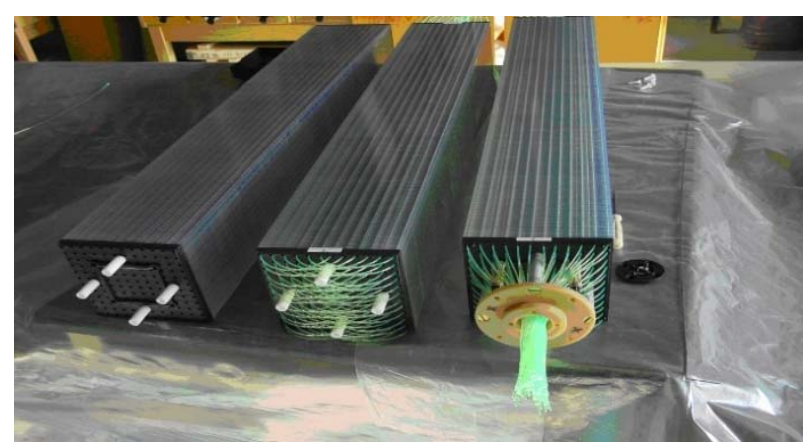

Fig. 6 General view of an improved calorimeter modules "Shashlyk" type and their constituent elements.

\section{Development of New Technologies}

3.1 The Technology of Melting of the Polystyrene Granules, Powdered with Scintillation Dopants in the Forms with Mirrors Walls

The design of mirror forms is very simple, it is a box with sides made of mirror-polished stainless steel plates. Scintillator manufactured in such form does not require machining and polishing. Several forms are manufactured for each scintillator dimension-type.

It was manufactured by this technology around 1,500 lengthy scintillation strips with dimensions of 15 $\times 100 \times 2,000 \mathrm{~mm}^{3}$ and $10 \times 60 \times 2,600 \mathrm{~mm}^{3}$ (Fig. 7) for Ghent University (Belgium), Universite Catholique De Louvain (Belgium) and University of Mainz (Germany). Four Y-11 type $1 \mathrm{~mm}$ diameter WLS-fibers assure 10 and 13 of PMT photoelectrons for MIP from the far end of the scintillator, respectively. 
Table 3 Physical characteristics (mold scintillators) (http://exwww.ihep.ru/scint/mold/product.htm).

\begin{tabular}{lllll}
\hline Type of a scintillator & $\begin{array}{l}\text { Light output from antracene } \\
(\%)\end{array}$ & $\begin{array}{l}\text { Time decay } \\
(\mathrm{ns})\end{array}$ & $\begin{array}{l}\text { Maximum of irradiation } \\
\text { spectra }(\mathrm{nm})\end{array}$ & Main applications \\
\hline SC-301 & 55 & 2.4 & 420 & $\beta, \gamma, \alpha$, fast $n$ \\
SC-302 & 57 & 2.3 & 430 & $\beta, \gamma, \alpha$, fast $n$ \\
SC-303 & 55 & 4.6 & 490 & $\beta, \gamma, \alpha$, fast $n$ \\
SC-304 & 57 & 2.5 & 420 & $\beta, \gamma, \alpha$, fast $n$ \\
SC-305 & 59 & 1.9 & 430 & For time of flight detectors \\
SC-306 & 66 & 2.3 & 418 & $\beta, \gamma, \alpha$, fast $n$ \\
SC-307 & 69 & 2.2 & 416 & $\beta, \gamma, \alpha$, fast $n$ \\
SC-308 & 69 & 2.2 & 416 & $\beta, \gamma, \alpha$, fast $n$ \\
SC-309 & 61 & 2.0 & 404 & $\beta, \gamma, \alpha$, fast $n$ \\
SC-321 & 46 & 2.5 & 430 & $4 \%$ Sn ${ }^{* *}$ for low energy $\gamma$ \\
SC-322 & 40 & 2.5 & 430 & $7 \%$ Pb $;$ for low energy $\gamma$ \\
SC-331 & 52 & 2.5 & 420 & $0.6 \% \mathrm{~B}^{10 * *} ;$ for $n$ of thermal energy \\
\hline
\end{tabular}

**The concentration of the dopes can be increased by request: $\mathrm{Sn}$, up to $8 \%$ weight fraction, $\mathrm{Pb}$, upto $12 \%$ weight fraction, $\mathrm{B}^{10}$, up to $5 \%$ weight fraction.

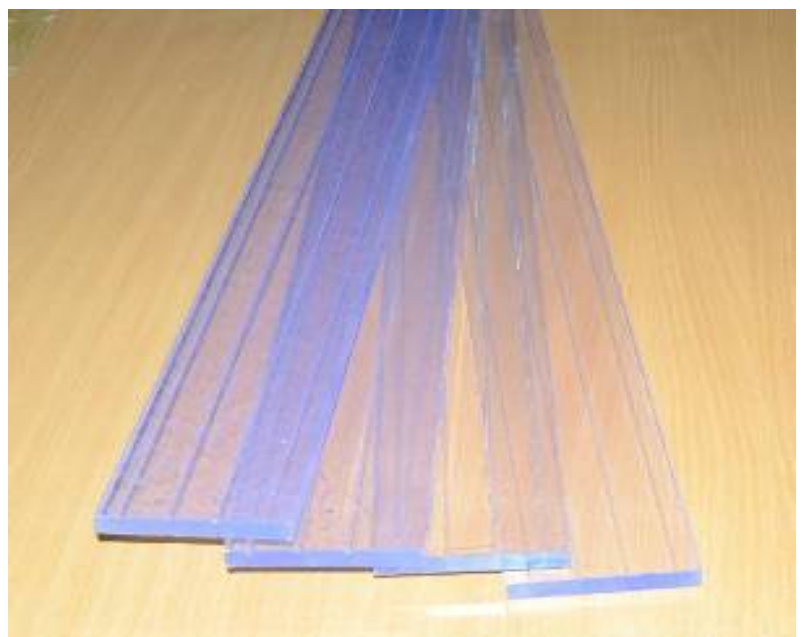

Fig. 7 The lengthy scintillation counters with dimensions $10 \times 60 \times 2,600 \mathrm{~mm}^{3}$ and grooves for WLS-fibers.

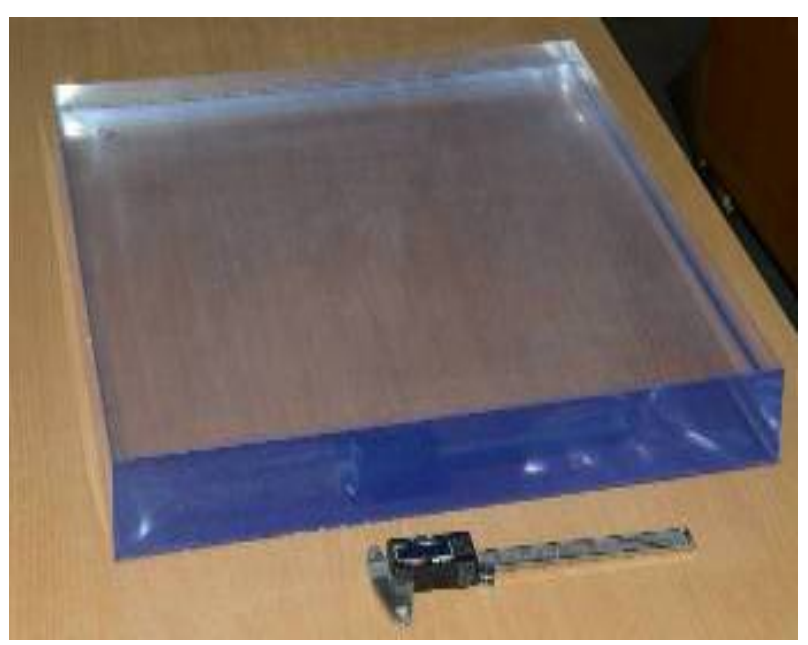

Fig. 8 The scintillation block with dimensions $50 \times 500 \times$ $500 \mathrm{~mm}^{3}$.
More than 1,000 scintillator blocks with dimensions $50 \times 500 \times 500 \mathrm{~mm}^{3}$ (Fig. 8) were supplied to the Baksan Neutrino Observatory. The light yield through the large face of the block from cosmic muon is $90 \%$ of the yield for polystyrene plastic scintillators, but 3 times lower cost.

As an example, Fig. 9 shows a solid scintillation counter with dimensions $30 \times 300 \times 1,500 \mathrm{~mm}^{3}$, made with the described technologies. For the light collection, the new manufactured at IHEP photomultiplier FEU-KS (Section 4) with the lengthy photocathode (15 $\times 200 \mathrm{~mm}^{2}$ ), is used.

The new PMT construction allows capturing without fiber scintillation light at the counter center with all its width. To improve the uniformity of the light collection along the length of the scintillation counter concentration of dopes varied from center to its ends. Number of photoelectrons (for MIP) from the ends was about 30 .

\subsection{The Scintillating Granules as Material for Ultra-Large Counters Manufacturing}

The conception of the new technology is to use WLS-fibers for light-collection system, run through the volume of the counter filled with scintillation granules ("bulk" counter) [24]. WLS-fibers of light collection system arranged periodically with optimal step. 


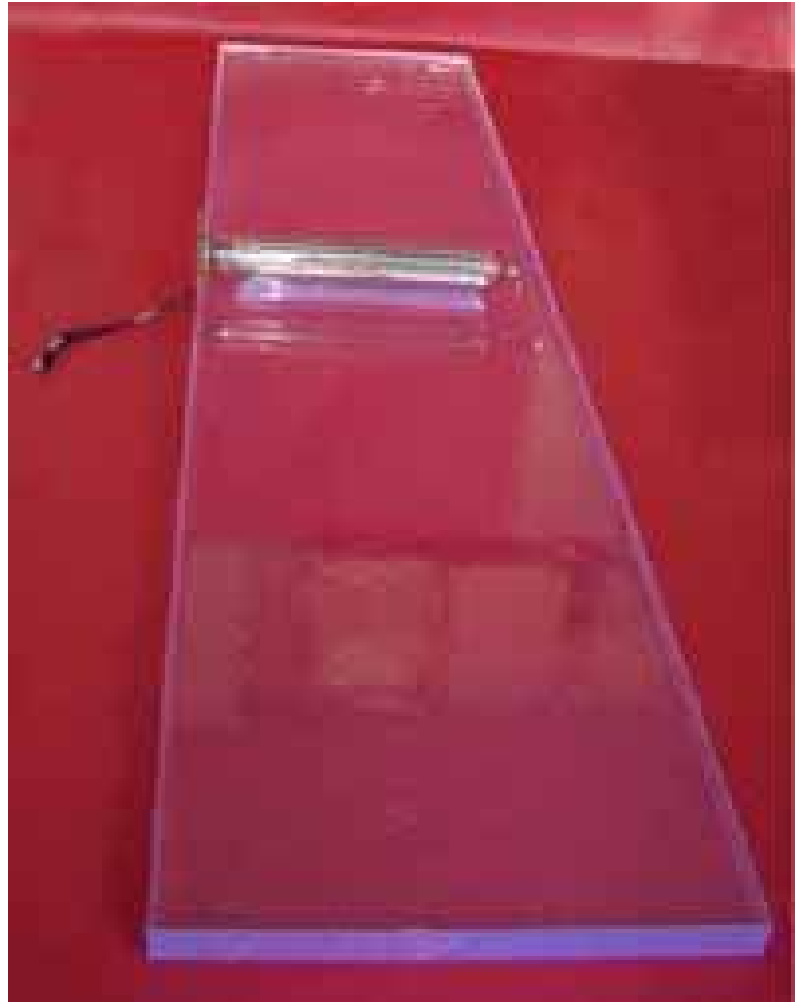

Fig. 9 The massive scintillation counter with dimensions 30 $\times 300 \times 1,500 \mathrm{~mm}^{3}$ made with the discussed technology. The light collection on photomultiplier FEU-KS with the lengthy photocathode.

Comparative measurements were made for light output from the scintillation counter with dimensions of $10 \times 140 \times 260 \mathrm{~mm}^{3}$ produced by polymerization (Fig. 10), and "bulk" type counter with the dimensions $20 \times 200 \times 200 \mathrm{~mm}^{3}$ (Fig. 11). In the last, one single row of periodically arranged WLS-fibers with $10 \mathrm{~mm}$ gap between them was used.

Scintillation counter volume was filled with $\varnothing 3 \times 3 \mathrm{~mm}$ granules, which ensures the effective density $0.65 \mathrm{~g} / \mathrm{cm}^{2}$. Measurements show that the PMT light output in the counter is about 40 ph.e./MeV.

Scintillating polystyrene granules can be produced on an industrial scale with a capacity of tens of thousands of tons per year, by using as a feedstock "powdered" granules of commercial transparent polystyrene.

The production output of a single industrial set-up for the production of polystyrene granules from styrene with scintillation dopes dissolved in the styrene can be hundreds of thousands tons per year. Scintillation dopants do not affect the manufacturing process.

\section{Thin Scintillation Counters Based on FEU-KS}

Several scintillation super-thin counters without light guide based on FEU-KS are presented in Fig. 12 (references above and Fig. 13).

Design of the new PMT allows to collect the scintillation light from the counters without light guide.

The PMT gain is more than $10^{6}$. The photocathode

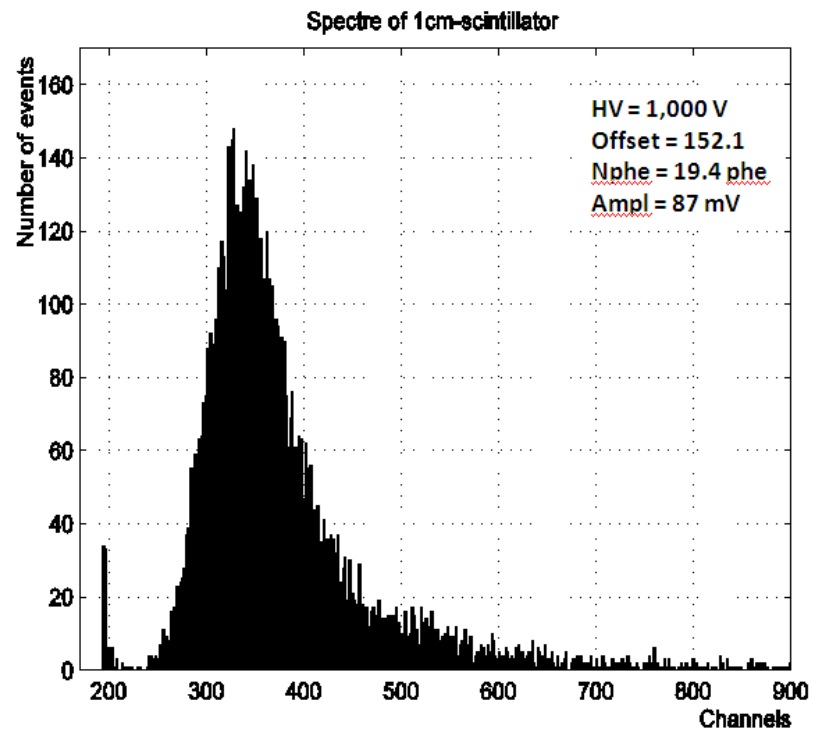

Fig. 10 Amplitude spectra from MIP from polymerized type scintillator with dimensions $10 \times 140 \times 260 \mathrm{~mm}^{3}$.

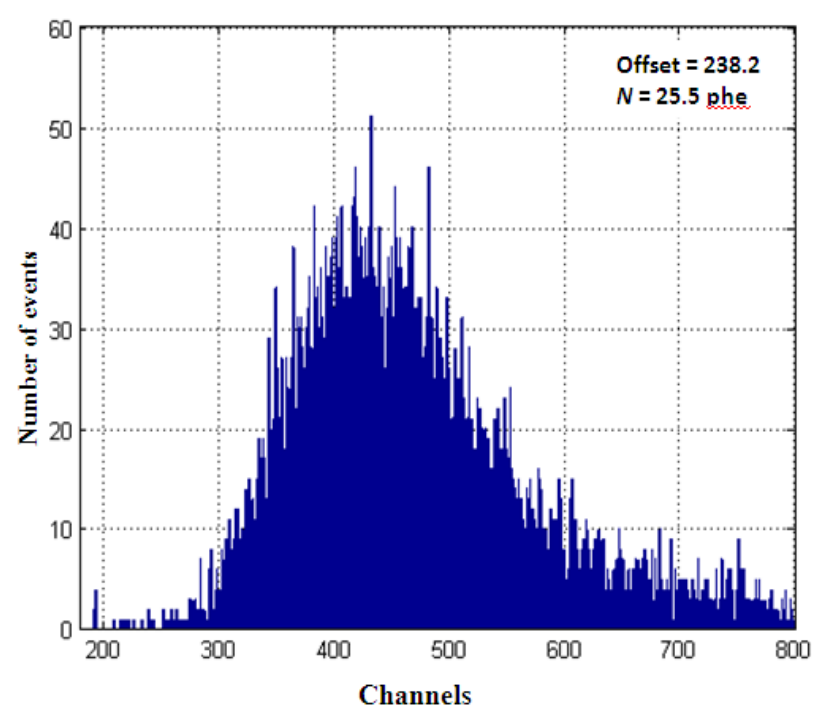

Fig. 11 Amplitude spectra from MIP from the "bulk" type counter with dimensions of $20 \times 200 \times 200 \mathrm{~mm}^{3}$. 


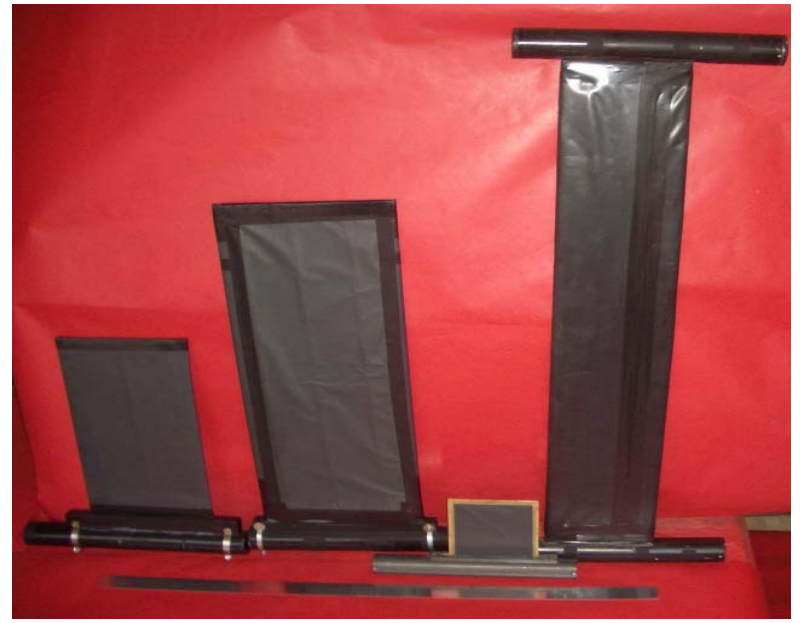

Fig. 12 Family of scintillation counters without light-guides based on FEU-KS with extended photocathode.

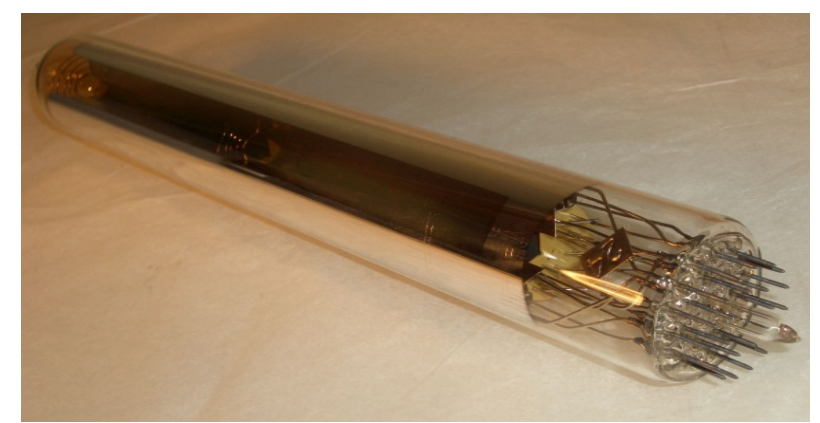

Fig. 13 New photomultiplier FEU-KS with an extended photocathode $\left(15 \times 200 \mathrm{~mm}^{2}\right)$, produced at IHEP.

blue sensitivity is up to $70 \mathrm{~mA} / \mathrm{W}$. The photoelectron number of thin polystyrene scintillators with sizes $1 \times$ $200 \times 200 \mathrm{~mm}^{3}$ and $0.5 \times 100 \times 100 \mathrm{~mm}^{3}$ are 20 and 12 per MIP, respectively.

\section{Conclusions}

In the near future, we are planning the following lines of investigation:

(1) Increasing the length of attenuation of light in the scintillators manufactured by extrusion, high-pressure molding under pressure and melting in the mirror forms to replace too expensive polymerized polystyrene scintillators;

(2) Investigation and optimization of scintillation granules manufacture and detectors on their basis;

(3) Development of "thin" counters for experiments at low energiesand ion beams;

(4) Increasing of radiation hardness of polystyrene scintillators.

\section{Acknowledgments}

The authors are gratefulto Zaitsev, A. M. and Soldatov, A. P. for the support of this work, Britvich, G. I. and Medinsky, M. V. for valuable comments, help with research and preparing the samples of scintillators. Work performed under the contract number N.4h.44.90.13.1118 from 31. 05. 2013.

\section{References}

[1] Alimova, T. V., Gorin, A. M., Ershova, T. A., Lapshin, V. G., Mekshun, P. A., Peresypkin, A. I., Rakhmatova, V. B., and Rykalin, V. I. 1986. "Properties of Scintillation Strips, Plates and Rods for Particle Counters, Manufactured by Extrusion." Preprint IHEP 35-86. (Serpukhov).

[2] Alde, D., Bellazzini, R., Binon, F. G., Boutemeur, M., Brez, A., Ditta, J., Donskov, S. V., Gouanere, M., Inaba, S., Kawai, H., Khaustov, G. V., Kinash, T., Knapp, E. A., Kobayashi, M., Lopez, T., Massai, M. M., Nakamura, T., Peigneux, J. P., Polyakov, V., Poulet, M., Rykalin, V. I., Samoylenko, V. D., Shagin, P. M., Singovsky, A. V., Stroot, J. P., Takamatsu, K., Torquati, M. R., and Tsuru, T. 1994. "The Slow Proton Position-Sensitive Time of Flight Detector of the GAMS NA 12/2 Experiment at CERN." Nuclear Instruments and Methods in Physics Research (NIM) A 342: 389-97.

[3] Belikov, S. V., Gurzhev, S. N., Gutnikov, Y. E., Lenisov, A. G., Kochetkov, V. I., Matveev, M. Y., Melnikov, E. A., and Usachev, A. P. 1995. "Scintillation Trigger System for Liquid Argon Neutrino Detector." Preprint IHEP 94-68 PTE 2: 43.

[4] Datsko, V. S., Donskov, S. V., Inyakin, A. V. 1987. "Investigations of the Energy and Spatial Resolution of the Modular Hadron Calorimeter at Energies of 18 and 38 GeV." Preprint IHEP 85-7. (Serpukhov)

[5] Vishnevsky, N. K., Vlasov, E. V., Gouz, Y. P., Dzhelyadin, R. I., Zaitsev, A. M., Lapin, V. V., Lapshich, V. K., Matveev, M. Y., Obraztsov, V. F., and Ostankov, A. P. 1994. "DELPHI Forward Muonhodoscope." Preprint IFVE-94-139. (Protvino)

[6] Alvsvaag, S. J. 1994. "The Silicon Shower Maximum Detector for the STIC, Contribution to 6 Pisa Meeting on Advanced Detectors." Presented at La Biodola, Isolad Elba, Italy.

[7] Adeva, B., Afanasyev, L., Benayoun, M., Benelli, A., Berka, Z., Brekhovskikh, V., and Caragheorgheopol, G. 2003. "DIRAC: A High Resolution Spectrometer for 
Pionium Detection." NIM A 515: 467-96.

[8] Semenov, V. K. 1986. "Large Tonnage Scintillator for Cellular Type Calorimeters." Presented at The IX All-Union Conference, Status and Prospects of the Development and Application of Scintillators and Scintillation Detectors in the 12th Five-Year Plan, Ukrainian Academy of Sciences, VNII Monocrystal, 86.

[9] Kadykov, M. G., Semenov, V. K., and Syzdalev, V. I. 1991. "Injection Molded Polystyrene Scintillator for Hadron Calorimeter." Instrum. Exp. Tech. (PTE) 34: $78-80$.

[10] Buyanov, V. M., Datsko, V. S., Pishchalnikov, Y. M., Semenov, V. K., and Skripachev, O. V. 1989. "Module of Electromagnetic Calorimeter Gepard-2000." Preprint IHEP-89-45. (Serpukhov)

[11] Bityukov, S. I., Semenov, V. K., and Yablokov. A. P. 1989. "Principal Characteristics of Cellular Type Hadron Calorimeter with Scintillator." Instrum. Exp. Tech. 32: 794-9.

[12] Gavrishchuk, O. P., Eremeev, R. V., Kadykov, M. G., Kukhtin, V. V., and Semenov, V. K. 1993. "Module of the Hadron Calorimeter HC-600.” Preprint JINR-P1-93-120. (Dubna 1993).

[13] Gavrishchuk, O. P., Kukhtin, V. V., Kuzmin, N. A., Manyakov, P. K., Maksimov, A. N., Mikhailov, Y. V., Savin, I. A., Semenov, V. K., Shalygin, A. B., and Vlasov, N. V. 2006. "Calorimeter for Detection of Hadrons in the Energy Range 10-GeV-100-GeV." Instrum. Exp. Tech. 49: 41-55.

[14] ATLAS Collaboration, Technical Proposal for a General Purpose pp Experiment at the Large Hadron Collider at CERN, CERN/LHCC/94-43, LHCC/P2 15 December 1994.

[15] Britvich, G. I., Chernichenko, S. K., Chubenko, A. P., Gilitsky, Y. V., Kushnirenko, A. E., Mamidzhanyan, E. A., Pavlyuchenko, V. P., Shein, I. V., Soldatov, A. P., Shepetov, A. L., and Vasil'chenko, V. G. 2006. "The Large Scintillation Charged Particles Detector of the
Tien-Shan Complex."ATHLE NIM A 564: 225-34.

[16] Britvich, G. I., Soldatov, A. P., Chernichenko, S. K., and Gilitsky, Y. V. 2009. Scintillation portal for detection of radioactive objects in traffic flows. Patent 86322 27.08.2009.

[17] Atoian, G. S., Gladyshev, V. A., Gninenko, S. N., Isakov, V. V., Kovzelev, A. V., and Monich, E. A. 1992. "Lead Scintillator Electromagnetic Calorimeter with Wavelength Shifting Fiber Readout." NIM A 320: 144-54.

[18] Atoian, G. S., Issakov, V. V., Karavichev, O. V., Karavicheva, T. L., Poblaguev, A. A., and Zeller, M. E. 2004. "Development of Shashlyk Calorimeter for KOPIO." NIM A 531: 467-80.

[19] Atoian, G. S., Britvich, G. I., Chernichenko, S. K., Dhawan, S., Issakov, V. V., and Karavichev, O. V. 2008. "An Improved Shashlyk Calorimeter." NIM A 584: 291-303.

[20] Dzhelyadin, R. 2009. "Recent Efforts in the Sampling Calorimetry.” NIM A 598: 229-34.

[21] Aphecetche Awes, T. C., Banning, J., Bathe, S., Bazilevsky, A., and Belikov, S. 2003. "PHENIX calorimeter." NIM. A 499: 521-36.

[22] Barsuk, S., Belikov, S. V., Denisov, A. G., Golutvin, A. I., Kochetkov, V. I., Mayatski, V. A., Novikov, E., Rusinov, D., Rusinov, V. Y., and Semenov, V. K. 2000. "The Facility to Control the Production of Shashlik Type Electromagnetic Calorimeter." LHCb-2000-044, CERN-LHCb-2000-044. 18.

[23] Hartouni, E. P., Van Apeldoorn, M. K. G., van der Graaf, H., Hamacher, W. R. T., Fan, R. S. X., Jiang, C., Schwitters R., Fan, X., Jiang, C., and Li, X. 1995. HERA-B: An Experiment to Study CP Violation in the B System Using an Internal Target at the HERA Proton Ring. Design Report, DESY-PRC-95-01, 491.

[24] Bitsadze, G. S., Joridze, V. P., Medinsky, M. V., and Rykalin, V. I., Scintillation Detector, Russian Federation Patent, 2511601, filed June 25, 2012, and issued April 10, 2014. 